\title{
International study of asthma and allergies in childhood (ISAAC): rationale and methods
}

\author{
M.I. Asher*, U. Keil**, H.R. Anderson***, R. Beasley+, J. Crane ${ }^{+}$, F. Martinez++, \\ E.A. Mitchell*, N. Pearce + B. Sibbald***, A.W. Stewart*, D. Strachan***, \\ S.K. Weiland**, H.C. Williams ${ }^{+++}$
}

International study of asthma and allergies in childhood (ISAAC): rationale and methods. M.I. Asher, U. Keil, H.R. Anderson, R. Beasley, J. Crane, F. Martinez, E.A. Mitchell, N. Pearce, B. Sibbald, A.W. Stewart, D. Strachan, S.K. Weiland, H.C. Williams. (CERS Journals Ltd 1995.

ABSTRACT: The aetiology of asthma and allergic disease remains poorly understood, despite considerable research. The International Study of Asthma and Allergies in Childhood (ISAAC), was founded to maximize the value of epidemiological research into asthma and allergic disease, by establishing a standardized methodology and facilitating international collaboration.

Its specific aims are: 1) to describe the prevalence and severity of asthma, rhinitis and eczema in children living in different centres, and to make comparisons within and between countries; 2) to obtain baseline measures for assessment of future trends in the prevalence and severity of these diseases; and 3 ) to provide a framework for further aetiological research into genetic, lifestyle, environmental, and medical care factors affecting these diseases.

The ISAAC design comprises three phases. Phase 1 uses core questionnaires designed to assess the prevalence and severity of asthma and allergic disease in defined populations. Phase 2 will investigate possible aetiological factors, particularly those suggested by the findings of Phase 1 . Phase 3 will be a repetition of Phase 1 to assess trends in prevalence.

Eur Respir J., 1995, 8, 483-491.
*Depts of Paediatrics \& Community Health, School of Medicine, University of Auckland, Auckland, New Zealand. **Institut für Epidemiologie und Sozialmedizin, Universität Münster, Münster, Germany. ***Depts of Public Health Sciences \& Primary Health Care, St George's Hospital Medical School, University of London, London, UK. +Dept of Medicine, School of Medicine, Wellington, New Zealand. ${ }^{++}$Dept of Paediatrics, Health Sciences Center, University of Arizona, Tucson, Arizona, USA. ${ }^{++}$Dept of Dermatology, Queens Medical Centre, University Hospital, Nottingham, UK.

Correspondence: M.I. Asher, Dept of Paediatrics, School of Medicine, University of Auckland, Private Bag 92019, Auckland, New Zealand.

Keywords: Allergies, asthma, childhood, eczema, rhinitis.

Received: May 191994

Accepted for publication December 291994

\section{Background}

There is considerable concern that the prevalence of asthma and allergic diseases is increasing in Western and developing countries. The International Study of Asthma and Allergies in Childhood (ISAAC) developed from the merging of two multinational collaborative projects, each investigating variations in childhood asthma at the population level. These projects were an initiative from Auckland, New Zealand to conduct an international comparative study of asthma severity, and an initiative from Bochum, Germany, in 1990, for an international study to monitor time trends and determinants of the prevalence of asthma and allergies in children. ISAAC has attracted worldwide interest and large scale participation.

\section{Aims and objectives}

The aims and objectives of the study are: 1) to describe the prevalence and severity of asthma, rhinitis and eczema in children living in different centres, and to make comparisons within and between countries; 2) to obtain baseline measures for assessment of future trends in the prevalence and severity of these diseases; and 3) to provide a framework for further aetiological research into lifestyle, environmental, genetic and medical care factors affecting these diseases.

\section{Asthma}

Asthma is one of the most important diseases of childhood, causing substantial morbidity [1-3]. Increases in the rates of hospital admission and primary care contacts for asthma in childhood $[4,5]$ have led to concern that the prevalence or severity of wheezing illness may be increasing in children. Trends in routine data are difficult to interpret because not all wheezy children receive a diagnosis of asthma, and the proportion who do has increased substantially over recent decades [6].

A number of surveys, first conducted in the 1960s and 1970 s, have been repeated in recent years using similar methodology, and these are consistent in showing an increase in the prevalence of wheezing illness (irrespective of diagnosis) in Britain, Australasia, Scandinavia, Israel and Taiwan, although the magnitude of the increase 
varies considerably between studies [7-17]. Few studies have been able to investigate trends in the severity of wheezing $[18,19]$, or changes in the pattern of health service utilization [15]. Only a few studies [10, 20] have included an objective assessment of bronchial reactivity in the same location at two points in time.

Geographical variations in the prevalence of wheezing illness have been found in Britain [21], Australia [22], and Germany [23, 24]. These are small by comparison with urban-rural differences described in southern Africa [25, 26]. Whilst genetic factors predispose to asthma, studies of children whose parents have migrated from developing to developed countries suggest that there is an increased risk of asthma associated with the environment or lifestyle of an industralized society [27, 28]. A further possibility is that some forms of asthma treatment might themselves be increasing asthma morbidity. There have been few international comparisons of prevalence using standardized methodology [29, 30], and none of these have included developing countries. The recent development of a standardized written and video questionnaire for self-completion by teenage children [31] offers scope for large scale studies of the prevalence and severity of wheezing illness in both developed and developing countries.

\section{Rhinitis}

There are no widely agreed criteria for the diagnosis or classification of rhinitis, and surprisingly little is known about its prevalence or distribution among children [32, 33]. The estimated prevalence of hay fever among school children in different countries has been reported to vary between 0.5 and $28 \%$ [34], but much of this variation is likely to be due to the diagnostic criteria and age group chosen. A few studies using a standardized case definition have suggested variations in prevalence within countries, by region or degree of urbanization [21, 24, $35,36]$.

Seasonal allergic rhinitis appears to have been exceedingly rare in Britain [37] and Switzerland [38] before the industrial revolution, and the prevalence of reported hayfever has increased in the United States [39], Sweden [9], and Britain [40] in recent decades. These trends may reflect changes in the perception and labelling of symptoms, or in presentation for medical diagnosis and treatment. Alternatively, they may reflect an increased propensity to allergic sensitization in successive generations, as suggested by two studies using skin prick tests to common aeroallergens at two points in time [41, 42].

\section{Eczema}

Little is known about the epidemiology of eczema (syn. atopic dermatitis, atopic eczema). However, geographical variations in the prevalence of eczema in children have been described in Britain, and these closely match the regional variations in hay fever $[21,35]$. Comparisons of parental report of eczema over time in Britain [40, 43], and examined eczema in Denmark [44], have sug- gested that eczema is more common amongst recent generations of children [45]. Studies of children whose parents have migrated from developing to developed countries suggest that eczema is associated with urbanization, and with development within those industralized countries [46, 47].

In theory, eczema is more readily confirmed by objective tests than either asthma or rhinitis. However, there are currently no internationally accepted criteria for defining atopic eczema in epidemiological surveys. A list of major and minor criteria proposed by HANIFIN and RAJKA [48] in the 1970s have been further evaluated [49] and widely applied in clinical studies, but have not been defined and standardized in a manner suitable for epidemiological studies. A team of British dermatologists and paediatricians has recently developed and validated definitions of atopic dermatitis based on questionnaire data with and without clinical signs [50-52]. These recommendations, which correspond closely to the major criteria proposed by HANIFIN and RAJKA [48], have been incorporated into the present study.

\section{Rationale of the ISAAC initiative}

Much research has been conducted into the reasons why some individuals rather than others develop asthma and other atopic diseases, such as rhinitis and eczema. A major risk factor is a family history of atopic disease, but various environmental factors are also considered important in the expression of disease. Such studies within populations have shed little light on the reasons why the occurrence of atopic disease varies from population to population. Factors affecting the prevalence of disease at a population level may be different to those that determine which individuals within a population are at greatest risk $[53,54]$. In addition, between populations the relationship between the three atopic conditions may be different. Ecological analyses between populations may reveal further important risk factors. One obstacle to the investigation of population differences (and of trends) has been the lack of a suitable and generally accepted method of measuring the prevalence and severity of asthma and other atopic diseases in children. Another obstacle has been the absence of a coordinated research programme to obtain and analyse comparative data. ISAAC has been developed to address these issues.

\section{Methods}

The collaborative studies will be conducted in three phases. Phase 1 uses core questionnaires designed to assess the prevalence and severity of asthma and allergic disease in defined populations. It is described here and in more detail in the ISAAC manual. In 1993, Phase 1 was completed in some centres; and others started/will start during 1994 and 1995. Phase 2 is being developed and will involve studies in informative centres of aetiological factors, particularly those suggested by Phase 1 . Phase 3 will be a repetition of Phase 1 , after at least 3 years. 


\section{Collaborating centres and registration}

Centres have been recruited from around the world through scientific networks, focusing on locations of particular interest. Each collaborating centre is responsible for completing a registration document and obtaining its own funding. Centres which provide a clean data set by the end of 1995 will be included in the initial worldwide data analysis.

\section{Selection of subjects}

The population of interest is school children within a specified geographical area (ISAAC Centre), of two age groups: 13-14 yrs and 6-7 yrs. The study sample will include all children within these age groups from a random sample of schools (cluster sampling, using schools as the sampling unit). A minimum of 10 schools (or all the schools) are needed to obtain a representative sample.

The older age group has been chosen to reflect the period when mortality from asthma is more common, and to enable the use of both a self-completed questionnaire and a video questionnaire. The younger age group has been chosen to give a reflection of the early childhood years, when asthma tends to be most prevalent, and hospital admission rates are higher.

\section{Sample size}

The aim is to have sufficient statistical power to detect differences of epidemiological and health service significance. Therefore, the sample size has been calculated with reference to more severe atopic disease rather than to the prevalence of disease of any severity. The sample size estimates are stringent because of the number of hypotheses being tested, the requirement to allow for intraschool correlation, and the need to have a high level of certainty in the results of such a major study.

A sample size of 3,000 has been chosen, which gives the following power for wheezing, rhinitis or eczema: if the true one year prevalence of wheezing is $30 \%$ in one centre and $25 \%$ in another centre, with the chosen sample size, the study power to detect this difference will be $95 \%$ at the $1 \%$ level of significance; if the true one year prevalence of severe symptoms is $5 \%$ in one centre and $3 \%$ in another centre, with the chosen sample size, the study power to detect this difference will be $90 \%$ at the $1 \%$ level of significance.

It is recognized that some centres may have limited resources or populations, but that it is, nevertheless, desirable for them to be included in the prevalence comparisons, providing that the sample size is at least 1,000 .

\section{Phase 1 core questionnaire modules}

Three one page questionnaires have been developed by the original collaborators (tables 1-6). These were agreed for use in the International Study of Asthma and Allergies in Childhood at a workshop in Bochum, Germany, 8-10 December, 1991. The aim of compiling a standardized "core" questionnaire is to ensure that comparable information on the basic epidemiology of atopic illnesses and their diagnosis is obtained from as many surveys as possible. The exact wording of questions follows, as far as possible, questions which have been used in published questionnaires and which have found differences between populations, and where validity has been assessed [31, 55-57].

The written core modules for 13-14 year olds on wheezing, rhinitis and eczema are used in all collaborating centres; the video questionnaire (see below) is strongly recommended. It is also strongly recommended to extend the study to 6-7 year olds using the appropriate written questionnaires on wheezing, rhinitis and eczema.

Brief questions about the child's age and gender are also included. Where comparisons between ethnic groups are planned, the question on ethnicity should preferably follow that used in the most recent Census of Populations in the individual centre.

A video questionnaire involving the audiovisual presentation of clinical symptoms and signs of asthma has been developed for 13 and 14 year old respondents [31, $56,57]$. This is described in detail elsewhere [56]; it is recommended to elicit information on wheezing and asthma without using language-dependent terminology. The feasibility of ISAAC Phase 1 written questionnaires and video questionnaire in 13-14 year olds has been demonstrated in a multicentre pilot study [31].

Responses to rhinitis questions (but not asthma or eczema questions) are affected by season (unpublished data from southern England and New Zealand). It is, therefore, recommended that at least half of the study population should be investigated before the main pollen season of the study area.

Translation of questionnaires from English into other languages will be standardized, in an attempt to define the best non-English version for each region, according to guidelines [58]. Each centre will need to obtain the necessary Ethics Committee approval prior to the start of the study.

\section{Additional modules}

It is anticipated that individual investigators may wish to supplement the core questions with questions of their own. The ISAAC Steering Committee has developed additional standardized questions about cough, and the medical care of asthma, rhinitis and eczema. Standardized protocols have also been developed for child contact instruments, including physical examination of the skin for flexural dermatitis [50-52], airway responsiveness testing using hypertonic saline aerosol challenge [59], skin prick tests for atopy, total and specific serum immunoglobulin E (IgE), and storage of blood samples for potential future genetic analyses. A compilation of all the ISAAC instruments will be published in 1995. 
Table 1. - Core questionnaire wheezing module for 13-14 year olds

1. Have you ever had wheezing or whistling in the chest at any time in the past?

Yes [ ] No [ ]

IF YOU ANSWERED "NO" PLEASE SKIP TO QUESTION 6

2. Have you had wheezing or whistling in the chest in the last 12 months?

Yes [ ] No [ ]

IF YOU ANSWERED "NO" PLEASE SKIP TO QUESTION 6

3. How many attacks of wheezing have you had in the last 12 months?

None [ ] 1 to 3 [ ] 4 to 12 [ ] More than 12 [ ]

4. In the last 12 months, how often, on average, has your sleep been disturbed due to wheezing? Never woken with wheezing [ ]

Less than one night per week [ ] One or more nights per week [ ]

5. In the last 12 months, has wheezing ever been severe enough to limit your speech to only one or two words at a time between breaths? Yes [ ] No [ ]

6. Have you ever had asthma? Yes [ ] No [ ]

7. In the last 12 months, has your chest sounded wheezy during or after exercise? Yes [ ] No [ ]

8. In the last 12 months, have you had a dry cough at night, apart from a cough associated with a cold or a chest infection? Yes [ ] No [ ]

Table 2. - Core questionnaire rhinitis module for 13-14 year olds

All questions are about problems which occur when you DO NOT have a cold or the flu.

1. Have you ever had a problem with sneezing, or a runny, or a blocked nose when you DID NOT have a cold or the flu?

Yes [ ] No [ ]

IF YOU ANSWERED "NO" PLEASE SKIP TO QUESTION 6

2. In the past 12 months, have you had a problem with sneezing, or a runny, or a blocked nose when you DID NOT have a cold or the flu? Yes [ ] No [ ]

\section{IF YOU ANSWERED "NO" PLEASE SKIP TO QUESTION 6}

3. In the past 12 months, has this nose problem been accompanied by itchy-watery eyes? Yes [ ] No [ ]

4. In which of the past 12 months did this nose problem occur? (please tick any which apply) January [ ] February [ ] March [ ] April [ ] May [ ] June [ ] July [ ] August [ ] September [ ] October [ ] November [ ] December [ ]

5. In the past 12 months, how much did this nose problem interfere with your daily activities? Not at all [ ] A little [ ]

A moderate amount [ ] A lot [ ]

6. Have you ever had hay fever? Yes [ ] No [ ] 
Table 3. - Core questionnaire eczema module for 13-14 year olds

1. Have you ever had an itchy rash which was coming and going for at least 6 months? Yes [ ] No [ ]

IF YOU ANSWERED "NO" PLEASE SKIP TO QUESTION 6

2. Have you had this itchy rash at any time in the last 12 months? Yes [ ] No [ ]

\section{IF YOU ANSWERED "NO" PLEASE SKIP TO QUESTION 6}

3. Has this itchy rash at any time affected any of the following places: the folds of the elbows, behind the knees, in front of the ankles, under the buttocks, or around the neck, ears or eyes? Yes [ ] No [ ]

4. Has this rash cleared completely at any time during the last 12 months?

Yes [ ] No [ ]

5. In the last 12 months, how often, on average, have you been kept awake at night by this itchy rash? Never in the last 12 months [ ]

Less than one night per week [ ] One or more nights per week [ ]

6. Have you ever had eczema? Yes [ ] No [ ]

Table 4. - Core questionnaire wheezing module for 6-7 year olds

1. Has your child ever had wheezing or whistling in the chest at any time in the past?

Yes [ ] No [ ]

IF YOU ANSWERED "NO" PLEASE SKIP TO QUESTION 6

2. Has your child had wheezing or whistling in the chest in the last 12 months?

Yes [ ] No [ ]

IF YOU ANSWERED "NO" PLEASE SKIP TO QUESTION 6

3. How many attacks of wheezing has your child had in the last 12 months?

None [ ] 1 to 3 [ ] 4 to 12 [ ] More than 12 [ ]

4. In the last 12 months, how often, on average, has your child's sleep been disturbed due to wheezing? Never woken with wheezing [ ]

Less than one night per week [ ] One or more nights per week [ ]

5. In the last 12 months, has wheezing ever been severe enough to limit your child's speech to only one or two words at a time between breaths? Yes [ ] No [ ]

6. Has your child ever had asthma? Yes [ ] No [ ]

7. In the last 12 months, has your child's chest sounded wheezy during or after exercise? Yes [ ] No [ ]

8. In the last 12 months, has your child had a dry cough at night, apart from a cough associated with a cold or a chest infection? Yes [ ] No [ ] 
Table 5. - Core questionnaire rhinitis module for 6-7 year olds

1. Has you child ever had a problem with sneezing, or a runny, or a blocked nose when he/she DID NOT have a cold or the flu? Yes [ ] No [ ]

\section{IF YOU ANSWERED "NO" PLEASE SKIP TO QUESTION 6}

2. In the past 12 months, has your child had a problem with sneezing, or a runny, or a blocked nose when he/she DID NOT have a cold or the flu?

Yes [ ] No [ ]

\section{IF YOU ANSWERED "NO" PLEASE SKIP TO QUESTION 6}

3. In the past 12 months, has this nose problem been accompanied by itchy-watery eyes? Yes [ ] No [ ]

4. In which of the past 12 months did this nose problem occur? (please tick any which apply) January [ ] February [ ] March [ ] April [ ] May [ ] June [ ]

July [ ] August [ ] September [ ] October [ ] November [ ] December [ ]

5. In the past 12 months, how much did this nose problem interfere with your child's daily activities?

Not at all [ ] A little [ ] A moderate amount [ ] A lot [ ]

6. Has your child ever had hay fever? Yes [ ] No [ ]

Table 6. - Core questionnaire eczema module for 6-7 year olds

1. Has your child ever had an itchy rash which was coming and going for at least 6 months? Yes [ ] No [ ]

IF YOU ANSWERED "NO" PLEASE SKIP TO QUESTION 7

2. Has your child had this itchy rash at any time in the last 12 months?

Yes [ ] No [ ]

IF YOU ANSWERED "NO" PLEASE SKIP TO QUESTION 7

3. Has this itchy rash at any time affected any of the following places: the folds of the elbows, behind the knees, in front of the ankles, under the buttocks, or around the neck, ears or eyes? Yes [ ] No [ ]

4. At what age did this itchy rash first occur? Under 2 years [ ]

Age 2-4 [ ] Age 5 or more [ ]

5. Has this rash cleared completely at any time during the last 12 months?

Yes [ ] No [ ]

6. In the last 12 months, how often, on average, has your child been kept awake at night by this itchy rash? Never in the last 12 months [ ]

Less than one night per week [ ] One or more nights per week [ ]

7. Has you child ever had eczema? Yes [ ] No [ ] 


\section{Data management}

The questionnaire completed by the parent/child is entered into the computer, exactly as recorded on the completed questionnaire. In general, each centre will be responsible for its own data entry, although in some regions/ countries one centre may take responsibility for this. The international comparison of the core data set will be performed in the International Data Centre (Auckland) with data transferred using the protocol described in the ISAAC Coding and Data Transfer Manual. The results of data analyses will be communicated to the ISAAC collaborators as information is produced, and further input on the data analyses will be sought from them. Each centre owns its own data.

Data from 6-7 year olds and 13-14 year olds will be analysed separately. Each measure of prevalence and severity will be compared between centres. The effect of cluster sampling by schools will be considered in the analysis.

Basic descriptive summaries of the data will be compiled and presented for each condition, in both age groups, for males and females, in each of the centres and countries participating. An ecological analysis will be undertaken using information on the geographical, environmental and ethnic characteristics of each centre. As well as the international comparisons, data will be analysed within each country. This will allow for the introduction of any additional variables that the country may have incorporated in the questionnaire.

\section{Plans for Phase 2}

The ISAAC Steering Committee is proposing a coordinated international study which will follow completion of ISAAC Phase 1 data analysis in 1995/1996. This next study, known as "ISAAC Phase 2 - International", will investigate variations in prevalence which emerge from Phase 1. Comparisons between populations (centres) will be undertaken, using objective measures of disease, and assessment of environment, lifestyle, and clinical management.

Populations which are potentially informative will be chosen, such as those with contrasting prevalence of disease, environmental exposures, management or genetic factors. Measurements on representative samples of these populations will be undertaken using the standardized ISAAC instruments (above). The sample sizes may be smaller than those recommended for Phase 1, and the age groups will be appropriate for the procedures to be undertaken. It is anticipated that small groups of centres in regional or national groups will plan local "ISAAC Phase 2" studies, encouraged by regional or national coordinators.

\section{Organization}

The organization of ISAAC consists of four levels: the Steering Committee, including the Executive; Regional Coordinators; National Coordinators; Collaborating Centres (fig. 1).

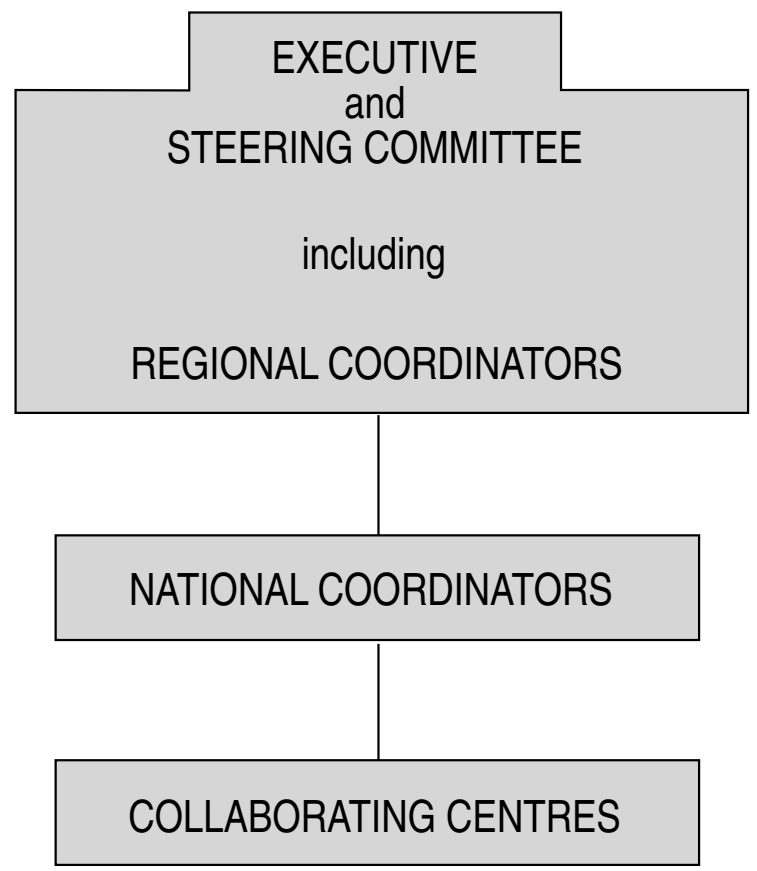

Fig. 1. - Organization of International Study of Asthma and Allergies in Childhood (ISAAC).

The Steering Committee. The responsibilities of the Steering Committee are to recruit regional coordinators; assist with the regional meetings; liaise with regional coordinators and provide assistance when required; coordinate the implementation and conduct of Phase 1; organize the further development of modules and methods for Phase 2; coordinate the analyses and publications of data; organize future international ISAAC meetings. The Steering Committee meets annually, but is coordinated on a day-to-day basis by a three member Executive.

Regional Coordinators. The regional coordinators are responsible for a broad region of the world. The regions are based on the six World Health Organization (WHO) regions of the world. However, in some instances, a WHO region may be split into smaller ISAAC regions, if the number of collaborating centres or countries is large, e.g. Europe has been divided into two subregions because of the large number of European countries participating.

National Coordinators. The national coordinators are generally responsible for a single country. However, in some instances, they may be responsible for several small neighbouring countries, particularly if these only have one collaborating centre and/or if no suitable national coordinators are available.

Collaborating Centres. The responsibilities of the collaborating centre are to complete the registration form; liaise with the national coordinator; carry out Phase 1 according to the protocol in the ISAAC manual; forward a "clean" data set to the national coordinator. 


\section{Appendix}

\section{ISAAC Steering Committee}

U. Keil'+ S. Weiland+ (Münster); E. von Mutius (München, Germany); M.I. Asher*+, E.A. Mitchell (Auckland); R. Beasley*, J. Crane, N. Pearce (Wellington, New Zealand); H.R. Anderson, D. Strachan* (London), B. Sibbald (Manchester), H. Williams (Nottingham), M. Burr (Cardiff) (UK); B. Björkstén ${ }^{+}$(Linköping, Sweden); F. Martinez ${ }^{+}$ (Tucson, USA); J. Mallol ${ }^{+}$(Santiago, Chile); G. Anabwani ${ }^{+}$ (Eldoret, Kenya); N. Ait-Khaled ${ }^{+}$(Algiers, Algeria); C. $\mathrm{Lai}^{+}$(New Territories, Hong Kong); J.R. Shah+ (Bombay, India); C. Robertson (Melbourne, Australia); S. Montefort ${ }^{+}$ (Malta). N.B. *: Executive; +: Regional Coordinator.

ISAAC documents available from Regional Coordinators: ISAAC Manual; ISAAC Coding and Data Transfer Manual; ISAAC Registration Document.

\section{References}

1. Gillam GL, McNichol KN, Williams HE. Chest deformity, residual airways obstruction and hyperinflation, and growth in children with asthma. Arch Dis Child 1970; 45: 789-799.

2. Anderson HR, Bailey PA, Cooper JS, Palmer JC, West S. Morbidity and school absence caused by asthma and wheezing illness. Arch Dis Child 1983; 58: $777-$ 784.

3. Anderson HR, Bland JM, Patel S, Peckham C. The natural history of asthma in childhood. $J$ Epidemiol Commun Health 1986; 40: 121-129.

4. Anderson HR. Increase in hospital admissions for childhood asthma: trends in referral, severity and readmissions from 1970 to 1985 in a health region of the United Kingdom. Thorax 1989; 44: 614-619.

5. Fleming DM, Crombie DL. Prevalence of asthma and hay fever in England and Wales. Br Med J 1987; 294: 279-283.

6. Anderson HR. Is asthma really increasing? Paediatr Respir Med 1993; 1: 6-10.

7. Turner KJ, Dowse GK, Stewart GA, Alpers MP. Studies on bronchial hyperreactivity, allergic responsiveness and asthma in rural and urban children of the highlands of Papua, New Guinea. J Allergy Clin Immunol 1986; 77: 558-566.

8. Hsieh KH, Shen JJ. Prevalence of childhood asthma in Taipei, Taiwan and other Asian Pacific countries. $J$ Asthma 1988; 25: 73-82.

9. Aberg N. Asthma and allergic rhinitis in Swedish conscripts. Clin Exp Allergy 1989; 19: 59-63.

10. Burr ML, Butland BK, King S, Vaughan-Williams E. Changes in asthma prevalence: two surveys 15 years apart. Arch Dis Child 1989; 64: 1118-1125.

11. Shaw RA, Crane J, O'Donnell TV, Porteous LE, Coleman ED. Increasing asthma prevalence in a rural New Zealand adolescent population: 1975-1989. Arch Dis Child 1990; 65: 1319-1323.

12. Burney PG, Chinn S, Rona RJ. Has the prevalence of asthma increased in children? Evidence from the national study of health and growth. Br Med J 1990; 300: 1306-1310.

13. Haahtela T, Lindholm H, Bjorkstein F, Koskenvuo K,
Laitenen LA. Prevalence of asthma in Finnish young men. Br Med J 1990; 301: 266-268.

14. Robertson CF, Heycock E, Bishop J, Nolan T, Olinsky A, Phelan PD. Prevalence of asthma in Melbourne school children: changes over 26 years. Br Med J 1991; 302: 1116-1118.

15. Strachan DP, Anderson HR. Trends in hospital admission rates for asthma in children. Br Med J 1992; 304: 819-820.

16. Laor A, Cohen L, Danon YL. Effects of time, sex, ethnic origin and area of residence on prevalence of asthma in Israeli adolescents. Br Med $J$ 1993; 307: 841-844.

17. Whincup PH, Cook DG, Strachan DP, Papacosta O. Time trends in respiratory symptoms in childhood over a 24 year period. Arch Dis Child 1993; 68: 729-734.

18. Anderson HR, Butland BK, Strachan DP. Trends in the prevalence and severity of childhood asthma. Br Med J 1994; 308: 1600-1604.

19. Weitzman M, Gortmaker SL, Sobol AM, Perrin JM. Recent trends in the prevalence and severity of childhood asthma. J Am Med Assoc 1992; 268: 2673-2677.

20. Peat JK, van den Berg RH, Green WF, Mellis CM, Leeder SR, Woolcock AJ. Changing prevalence of asthma in Australian children 1982-1992. Br Med J 1994; 308: 1591-1596.

21. Strachan DP, Golding J, Anderson HR. Regional variations in wheezing illness in British children: effect of migration during early childhood. J Epidemiol Commun Health 1990; 44: 231-236.

22. Salome CM, Peat JK, Britton WJ, Woolcock AJ. Bronchial hyperresponsiveness in two populations of Australian school children. I. Relation to respiratory symptoms and diagnosed asthma. Clin Allergy 1987; 17: 271281.

23. von Mutius E, Fritzch Ch, Weiland SK, Roll G, Magnussen $\mathrm{H}$. Prevalence of asthma and allergic disorders among children in the united Germany: a descriptive comparison. Br Med J 1992; 305: 1395-1399.

24. Weiland SK, Mundt KA, Ruckmann A, Keil U. Selfreported wheezing and allergic rhinitis in children and traffic density on street of residence. Ann Epidemiol 1994; 4: 243-247.

25. van Niekerk CH, Weinberg EG, Shore SC, Heese H de V, van Schalkwyk DJ. Prevalence of asthma: a comparative study of urban and rural Xhosa children. Clin Allergy 1979; 9: 319-324.

26. Keeley DJ, Neill P, Gallivan S. Comparison of the prevalence of reversible airways obstruction in rural and urban Zimbabwean children. Thorax 1991; 46: 549553.

27. Waite DA, Eyles EF, Tonkin SL, O'Donnell TV. Asthma prevalence in Tokelauan children in two environments. Clin Allergy 1980; 10: 71-75.

28. Morrison Smith J, Harding LK, Cumming G. The changing prevalence of asthma in school children. Clin Allergy 1971; 57-61.

29. Asher MI, Pattemore PK, Harrison AC, et al. International comparison of the prevalence of asthma symptoms and bronchial hyperresponsiveness. Am Rev Respir Dis 1988; 138: 524-529.

30. Barry DMJ, Burr ML, Limb ES. Prevalence of asthma among 12 year old children in New Zealand and South Wales: a comparative survey. Thorax 1991; 46: 405409.

31. Pearce N, Weiland SK, Keil U, et al. Self-reported prevalence of asthma symptoms in children in Australia, England, Germany and New Zealand: an international 
comparison using the ISAAC protocol. Eur Respir $J$ 1993; 6: 1455-1461.

32. Sibbald B, Rink E. Epidemiology of seasonal and perennial rhinitis: clinical presentation and medical history. Thorax 1991; 46: 895-901.

33. Sibbald B, Strachan D. Epidemiology of rhinitis. In: Busse W, Holgate ST, eds. Mechanisms in asthma and rhinitis: implications for diagnosis and treatment. Oxford, Blackwell Scientific Publications, 1994; pp. 32-43.

34. Montgomery-Smith J. Epidemiology and natural history of asthma, allergic rhinitis and atopic dermatitis (eczema). In: Middleton E, Reed CE, Ellis EF, Adkinson NF, Yunginger JW, eds. Allergy: Principles and Practice. St Louis, C.V. Mosby, 1983; pp. 771-803.

35. Golding J, Peters T. Eczema and hayfever. In: Butler NR, Golding J, eds. From Birth to Five: A Study of the Health and Behaviour of Britain's Five Year Olds. Oxford, Pergamon, 1986; pp. 171-186.

36. Britton WJ, Woolcock AJ, Peat JK, Sedgwick CJ, Lloyd DM, Leeder SR. Prevalence of bronchial hyperresponsiveness in children: the relationship between asthma and skin reactivity to allergens in two communities. Int J Epidemiol 1986; 15: 202-209.

37. Emmanuel MB. Hay fever, a post industrial revolution epidemic: a history of its growth during the 19th century. Clin Allergy 1988; 18: 295-304.

38. Wuthrich B. Epidemiology of allergic diseases: are they really on the increase? Int Arch Allergy Appl Immunol 1989; 90: 3-10.

39. Hagy GW, Settipane GA. Bronchial asthma, allergic rhinitis and allergy skin tests among college students. $J$ Allergy 1969; 44: 323-332.

40. Ninan TK, Russell G. Respiratory symptoms and atopy in Aberdeen schoolchildren: evidence from two surveys 25 years apart. $B r$ Med $J$ 1992; 304: 873-875.

41. Barbee R, Kaltenborn W, Lebowitz M, Burrows B. Longitudinal changes in allergen skin test reactivity in a community population sample. J Allergy Clin Immunol 1987; 79: 16-24.

42. Sibbald B, Rink E, D'Souza M. Is atopy increasing? $\mathrm{Br}$ J Gen Pract 1990; 40: 338-340.

43. Taylor B, Wadsworth M, Wadsworth J, Peckham C. Changes in the reported prevalence of childhood eczema since the 1939-1945 war. Lancet 1984; ii: 1255-1257.

44. Schulz-Larsen F, Holm NV, Henningsen K. Atopic dermatitis. A genetic-epidemiological study in a populationbased twin sample. J Am Acad Dermatol 1986; 15: 487-494.

45. Williams HC. Is the prevalence of atopic dermatitis increasing? Clin Exp Dermatol 1992; 17: 385-391.

46. Worth RM. Atopic dermatitis among Chinese infants in
Honolulu and San Francisco. Hawaii Med J 1962; 22: 31-35.

47. Williams HC, Pembroke AC, Forsdyke H, Boodoo G, Hay RJ, Burney PGJ. London-born black Caribbean children are at increased risk of atopic dermatitis. $\mathrm{J} \mathrm{Am}$ Acad Dermatol 1995; 32: 212-217.

48. Hanifin JM, Rajka G. Diagnostic features of atopic dermatitis. Acta Derm Venereol (Stockholm) 1980; 92: 44-47.

49. Diepgen TL, Fartasch M, Hornstein OP. Evaluation and relevance of atopic basic and minor features in patients with atopic dermatitis and in the general population. Acta Derm Venereol (Stockholm) 1989; Suppl. 144: 50 54.

50. Williams HC, Burney PGJ, Hay RJ, et al. The UK Working Party's diagnostic criteria for atopic dermatitis. I. Derivation of a minimum set of discriminators for atopic dermatitis. Br J Dermatol 1994; 131: 383-396.

51. Williams HC, Burney PGJ, Strachan D, Hay RJ. The UK Working Party's diagnostic criteria for atopic dermatitis. II. Observer variation of clinical diagnosis and signs of atopic dermatitis. Br J Dermatol 1994; 131: 397405.

52. Williams HC, Burney PGJ, Pembroke AC, Hay RJ. The UK Working Party's diagnostic criteria for atopic dermatitis. III. Independent hospital validation. $\mathrm{Br} J$ Dermatol 1994; 131: 406-416.

53. Rose G. Sick individuals and sick populations. Int $J$ Epidemiol 1985; 14: 32-38.

54. Rose G. The strategy of preventive medicine. Oxford University Press, 1992.

55. Burney PGJ, Laitinen LA, Perdrizet S, et al. Validity and repeatability of the IUATLD (1984) Bronchial Symptoms Questionnaire: an international comparison. Eur Respir J 1989; 2: 940-945.

56. Shaw RA, Crane J, Pearce N, et al. Comparison of a video questionnaire with the IUATLD written questionnaire for measuring asthma prevalence. Clin Exp Allergy 1992; 22: 561-568.

57. Shaw RA, Crane J, O'Donnell TV, Lewis ME, Stewart $\mathrm{B}$, Beasley R. The use of a video-taped questionnaire for studying asthma prevalence: a pilot study among New Zealand adolescents. Med J Aust 1992; 157: 311314.

58. Weiland SK, Kugler J, von Mutius E, et al. The language of pediatric asthma patients: verbal descriptors of symptoms in Germany. Monatsschr Kinderheilkd 1993; 141: 878-882 (in German).

59. Riedler J, Reade T, Robertson CF. Repeatability of the response to $4.5 \% \mathrm{NaCl}$ challenge in children with mild to severe asthma. Pediatr Pulmonol 1994; 18: 330-336. 